\title{
The Internet: \\ A Selective Annotated Bibliography \\ of Print Material
}

\author{
by Marlene Giguère
}

\begin{abstract}
Since the Internet is such an ideal medium for electronic publishing, much of the information about it is available in that format. This can be a problem for the Internet beginner who does not have access to the network or who does not know how to retrieve information in an electronic format. The objective of this annotated bibliography, therefore, is to provide a selection of items available in print which the novice may consult before accessing or using the Internet. Material selected is introductory in nature. Topics covered include: guides and directories to resources, general information about the Internet and its three main applications(electronic mail, remote login and file transfer) as well as information about tools used on the Internet to facilitate locating information.
\end{abstract}

\section{INTRODUCTION}

The Internet originated as a specialized research network to facilitate scientific and military communication among a select group of computer literate users. However, it quickly expanded into the research and academic communities and now the commercial sector is one of the fastest growing ones on the Internet today. Hosts available on the Internet have increased from 235 to 992 thousand from 1982 to 1992. (Marine, 1992, p. 132) Estimates on the number of users range as high as 10 million with 10 percent growth per month (LaQuey, 1993, p. 6).

However, despite its phenomenal growth and popularity, the Internet still remains a relatively obscure and unfriendly environment for the uninitiated. This can, no doubt, be attributed to its origins within a specialized user community, its rapid development and its flexibility to network with a number of different systems. Given this fact, the need for information on how to successfully use the Internet is a particularily important issue. The growing body of users and potential users require information about what the Internet is and its utility, how it can be of use to them, and how it can be accessed and effectively used before becoming committed users. There is a growing body of literature about Internet which is characterized by two main features : recency and nontraditional publishing format.

Unlike more traditional library topics with histories extending over a number of years or even decades, Internet is a new phenomenon. It was only in the mid-nineteen eighties that the Internet (as we know it now) began its evolution from a highly technical and experimental network to one accessible to the research and educational communities, and as a result to the library community as well. The literature on the topic is, therefore, a reflection of this development, with few publications of interest to librarians appearing until the end of the eighties. There have been few overviews of the literature, given the relative recency of its importance to the library community.
Secondly, the Internet is an ideal environment for electronic publishing because information can be rapidly written, updated and distributed to a large number of users. It is therefore logical that much of the information about the Internet (how to use it, its value, etc.) is available in an electronic format on the Internet. Accessing this information, however, can be a problem for the novice user who requires basic print documentation as the first step in understanding the wealth of electronic material available.

The objective of this bibliography, therefore, is to provide an introductory selection of the print literature currently available on the topic. As a preliminary tool for new Internet users, it can serve as a pointer to print literature on general and specific aspects of interest to new Internet users.

\section{COVERAGE}

This bibliography covers introductory material dealing with the Internet, its three major applications, (electronic mail, remote login and downloading of texts and software) and tools designed to assist in its exploitation. Coverage includes material published until the first quarter of 1993.

Selection of titles was based on the following criteria:

\section{Available in print format.}

This is true with the exception of two items which are cited because of their importance. They should be fairly easy to acquire from major libraries in a print format because they provide informationaboutaccessing online publicaccess catalogs. (In each case further information is given within the specific annotation.) 


\section{Introductory in nature.}

Material of an advanced or technical nature was not included due to the introductory nature of the bibliography.

\section{Contributing to a general or global understanding of the Internet or one of its applications or tools.}

Material dealing with a specific institution, with a specific system, or with a particular use of an application was excluded unless it enhanced general understanding of the topic. Some items, such as articles about bulletin boards of interest to librarians, were retained because of their particular interest to the readership.

\section{ARRANGEMENT}

The bibliography is arranged in five main sections according to the following categories:

\section{Guides}

The Internet has been described as a highway, an ocean and even a dwelling. (Tennant, Ober and Lipow, 1993, p. 9). Regardless of whether you are travelling it, surfing it, or moving into it, a guide is essential for exploring the Internet. Guides provide overviews as well as basic descriptions and advice on how to effectively use the resources available on the Internet. They are of particular importance to the first time user and are recommended as one of the first purchases to be made.

\section{Directories}

Because the Internet has evolved in a dynamic, random and decentralized manner, there is no one institution responsible for organizing all of the information available on the Internet. As a result, certain organizations or individuals have assumed the responsibility of creating inventories or directories of informationfor a particular application or activity. This section has been divided into several subsections to reflect this fact. There are subsections for the following categories: OPACs (online public access catalogs), electronic discussion groups, and electronic mail addresses.

Used in conjunction with guides, these specialized directories can assist in locating a specific item of information, such as an address or a title amidst the voluminous amount of information available on the Internet. Directories are tools which enhance a user's effectiveness on the network.

\section{General information}

First-time users may simply wish to browse the literature or read brief overviews of what the Internet has to offer. This third section is devoted to items of general interest which have been selected from a variety of titles, with the view that the typical reader will have access to some, if not all, items suggested. Sample topics include an explanation of the global environment in which the Internet is situated, Internet applications within the library community and policy issues yet to be resolved.

\section{Internet applications}

Internet is used by many communities, scientific, academic, business, etc. It has a number of uses, however, there are three main applications of interest to the library community. In order to facilitate consultation of this section of the bibliography, it has been divided into three sections to represent each of these applications.

The first application, electronic mail, is a facility which transmits messages electronically to an individual or a group on the network. This is an extremely popular feature because messages can be sent or received on a local, national or international basis (provided both users are on connecting networks) and consulted at the user's convenience. Although originally used to send messages between users, the potential of this application soon became evident and developed into a means of communicating with a number of individuals simultaneously. This explains the rise of electronic discussion groups which are used for professional and recreational purposes to communicate with a group of users with similar interests. As a further means of communicating with their membership, discussion groups have now created electronic serials, in the form of newsletters and journals for more formal and rapid transmission of information. The e-mail application on Internet now provides access to individuals, to groups and to electronic publications.

Thesecond use of the Internet is its remote login application. This allows users to connect with remote computers and consult their resources much as one does when conducting an online search. In fact many of the database vendors, such as Dialog and Mead, are accessible on the Internet using this application. In the library community this application is frequently employed to search the hundreds of library online public access catalogs (OPACs) accessible on the Internet.

The final application allows for retrieval of large text files or software from computers across the network. Because these computers make this information freely available, users do not require authorization and can access the information by logging in as "guest" or "anonymous" and entering their e-mail address as the password. This is the reason that it is frequently referred to as an anonymous FTP (File Transfer Program).

\section{Tools on the Internet}

A number of tools have been designed to facilitate access to resources on the network. Although some might argue that these tools are usually used by more experienced users, they can be advantageous to the new user by guiding them to specific resources and thereby reducing time and frustration. For this reason, this final section has been divided into three subsections to represent three typical tools. These are known as Archie (Archive without the letter ' $v$ '), Gopher (the animal with burrowing instincts) and WAIS (Wide Area Information Server). Each one helps users to find information on the Internet in a different way and they can be complementary in nature. They are, however, only a representative sampling of a number of such tools available currently. Readers may wish to explore others which may better suit their particular needs. As a final note, items 
included in this section will be of interest to those following trends in managing and retrieving electronic information.

\section{SOURCES}

This bibliography was compiled based on searches conducted in the following databases: ERIC, LISA, and Information Science Abstracts. In addition, Magazine Index, Computer Database, INSPEC, and NTIS were scanned for appropriate introductory material.

\section{NOTE}

This bibliography is produced according to the Publication Manual of the American Psychological Association. In two cases, the user will note that the traditional bibliographic citation is followed by further information enclosed in square brackets. This information is provided because the document is available in an electronic format. It gives the address and directory location where the user can locate the material and import it using the file transfer or electronic mail applications on the Internet. Readers who wish to obtain these files should refer to items included in the appropriate section (e-mail or FTP) for further information before retrieving files.

\section{GUIDES}

Kehoe, Brendan P. (1993). Zen and the art of the Internet : A beginner's guide (2nd ed.). EnglewoodCliffs, NJ: PrenticeHall.

Written for the novice user, this guide provides simple, nontechnical chapters explaining the basic applications available on Internet (e-mail, file transfer and remote login). It also includes a chapter on Usenet News, a popular electronic discussion group service, as well as descriptions of various tools and commercial services available on the network. Designed to be an introductory guide and relerence work, it fulfills both purposes. Interspersed with interesting anecdotes this is a highly readable text. Includes glossary, bibliography and an index.

Krol, Ed. (1992). The whole Internet: User's guide \& catalog. Sebastopol, CA: O’Reilly.

Previously published in electronic format as "The Hitchhiker's Guide to the Internet", this completely revised and updated print copy has been widely and very well received. It is a detailed and comprehensive guide which covers the basics through to the current tools available to help users find people, software and other resources. Useful for novice users, it is equally helpful at an intermediary level as a reference and resource guide. It is supplemented with a user catalog listing resources available on the Internet on subjects as diverse as aeronautics to zymurgy (home brewing)! In addition to the usual glossary and list of service providers, this attractively illustrated guide comes with a handy quick reference card.

Lane, Elizabeth and Summerhill, Craig. (1993). Internet primer for information professionals: A basic guide to Internet networking technology. Westport, CT: Meckler.

This guide will appeal to those interested in acquiring a basic understanding of the technology involved. In addition to explanations of Internet and its main applications, it explains the different levels of connectivity to the Internet, which is important information for first time users. It also covers the basics of protocols and file formats. A final chapter is devoted to some unresolved issues, such as future funding of the network, copyright and security concerns and the inclusion of the private sector. Includes bibliography.

LaQuey, Tracy. (1993). The Internet companion: A beginner's guide to globalnetworking. Reading, MA:Addison-Wesley.

This pocket-sized volume covers all the basic information required for accessing and using the Internet. It is divided into highly readable and understandable sections with a number of illustrations and short vignettes. Includes a bibliography and extensive appendix on Internet resources.

Marine, April (Ed.). (1992). Internet: Getting started. Menlo Park, CA: SRI International.

As the first volume in the Internet Information Series, this title's "primary purpose is to tell you how to join the Internet." Part I focuses on providing information about accessing the Internet. The different types of access available with their costs are explained. Names and essential information about Internet service providers is organized geographically by state or country. This information is also available in an alphabetic arrangement in an appendix. This section of the volume dealing with service providers is particularly important for readers who do not currently have access to the Internet. Part II is an introduction to the Internet and covers basic concepts, descriptions of the three main applications, resources available and descriptions of Internet organizations on a national and international scale.

Tennant, Roy, Ober, John, and Lipow, Anne. (1993). Crossing the Internet threshold: an instructional handbook. Berkeley, CA: Library Solutions Press.

In the foreword, Clifford Lynch, Director of Library Automation, University of California, describes this work as covering "the issues faced by ...new users, and even users-to-be trying to find out how to get connected." In addition to standard chapters on the basic Internet applications, it also includes succinct fact sheets on essential Internet services and tools, like Usenet, Archie, Gopher, etc. This spiral bound handbook, which evolved out of a two day workshop, is unique because it includes exercises 
for beginners through to advanced users. Its vocation is also unique in that it is designed for two categories of users: new users and trainers. For those who would eventually like to instruct others on the use of the Interisit, this item is particularly interesting. It includes: a course outline, overheads, course evaluation forms, and a ckecklist for trainers. Includes glossary, bibliography and information on connection.

\section{DIRECTOR!ES}

\section{Directories of online public access catalogs}

Barron, Billy. (1992). UNT's accessing on-line bibliographic databases. [Available by anonymous FTP from host ftp.unt.edu, directory library, filenames libraries.ps, libraries.txt, libraries.con, libraries.adr, libraries.wp]

This directory of online public access catalogs is international in scope. Its arrangement is alphabetical by name of institution. Each OPAC entry is standardized to include logon and exit instructions for the library catalog. In many cases there is a reference within the entry to an appropriate appendix which provides basic explanations on how to use the library system in that specific institution. Examples of systems covered in appendices are NOTIS, GEAC, Dynix, among others. This directory is updated periodically. Includes brief bibliography.

NOTE: This directory is currently not available in print. However, major universities often distribute print copies for their user population.

Henry, Marcia, Keenan, Linda \& Reagan, Michael. (1991). Search sheets for OPACs on the Internet: A selective guide to U.S. OPACs utilizing VT100 emulation. Westport, CT: Meckler.

This title is recommended for use with the directories in this section. A major problem with searching OPACs on the Internet is the number of different OPAC systems each with its own specific command language. While Internet can easily position a user in an OPAC, the user must understand that particular OPAC's system commands in order to search it efficiently. For users scanning a number of library catalogs this means that they may need to use several different sets of commands. This guide provides a partial solution to this problem. Twenty five major OPAC systems are described in "standardized search sheets." These fact sheets contain commands for searching, displaying, editing, and calling up help screens. This guide is limited to only one type of terminal emulation which is the VT100.

St. George, Art and Larsen, Ron. (1992). Internet-accessible library catalogs and databases. [Available by e-mail message “GET LIBRARY PACKAGE” to listserv@unmvm.bitnet]

Also international in scope, this OPAC directory has sections arranged by state or country for the following types of resources: catalogs \& databases accessible without charge, those accessible for a charge, intemational catalogs, dial-up libraries and catalogs, a list of bulletin boards arranged by subject, American and international CWISs (Campus-Wide Information Systems) and a list of resource directories. Generally listings provide network addresses, basic logon instructions, descriptions of holdings and names of contact people.

NOTE: This directory is currently not available in print although a sample section can be found in the Directory of computer conferencing in libraries. Major universities often distribute print copies for their user population.

\section{Directories of electronic discussion groups}

Hardie, Edward and Neon, Vivian (Eds.) (1992) Internet: Mailing lists. Menlo Park, CA: SRI International.

This second volume in the Internet Information Series provides an introduction to the rich and varied world of discussion groups active on the Internet. Over 700 groups are described with complete subscription information. Discussion group subjects cover technical, professional and leisure interests.

Okerson, Ann (Ed.). (1992). The directory of electronic journals, newsletters and academic discussion lists (2nd ed). Compiled by Michael Strangelove and Diane Kovacs. Washington: Association of Research Libraries.

This publication is composed of 2 separate listings: Part I, Strangelove's Directory of Electronic Journals and Newsletters and Part II, Kovacs' Academic Discussion Lists and Interest Groups. Part I is further divided into two sections, one dealing withelectronic journals and the otherwith electronic newsletters. Each section is arranged alphabetically by title. Entries provide a brief synopsis of each item's philosophy, its contents, and subscription information. Part II has a different organization. It presents discussion groups according to a broad subject classification (humanities, physical sciences, etc.). Coverage is limited to groups with a scholarly or academic vocation. Basic information for subscription is included.

\section{Williams, Brian. (1992). Directory of computer conferencing in libraries. Westport, CT: Meckler Publishing.}

In explaining what a computer conference is, the author states that there is "... a continuum of electronic computer-based communication from electronic mail through bulletin boards to computer conferencing." As described in this volume, a computer conference is a more sophisticated type of bulletin board service. This work discusses networks, system software used to support conferences, and individual conferencing systems. Descriptions of specific systems and conferences are supported with sample sessions. For example, LITA (Library and Technology Association) is one of 14 conferencing systems profiled. This profile includes a brief description, access information, charges, special features and a typical session. A significant portion of the work is devoted to appendices such as the reproduction of a 
sample section of the Internet-Accessible Library Catalogs and Databases, a list of USENET groups and a list of BITNET discussion groups among others. Includes extensive bibliography.

\section{Directories of electronic mail addresses}

Frey, Donnalyn and Adams, Rick. (1990). !\%@:: A directory of electronic mail addressing and networks. 2nd edition. Sebastopol, CA.: O’Reilly.

Currently there is no one directory which contains the electronic mail addresses for all Internet users. This directory/ handbook provides an introduction to electronic mail as well as fact sheets for individual networks which graphically illustrate the geographical range of each network and its connections. This volume is comprehensive in that it covers 130 networks internationally, some of which are part of or communicate with the Internet and some of which are separate. This item is useful for obtaining information on how to navigate between networks, for locating information about a specific network and for obtaining addresses by country, by organization, etc.

\section{GENERAL INFORMATION}

Arms, Caroline R. (1990). A new information infrastructure. Online 14, 15-22.

For those interested in a global understanding of how this "new information infrastructure" has and will continue to effect the library environment, this classic article is recommended. The first part of the article is devoted to explaining what a network is, how Internet developed and how it compares with BITNET. The second part is a discussion of future developments such as how Internet will affect the library of the future. Includes a sidebar entitled What's out there? A network resource guide and a short bibliography.

Arms, Caroline R. (1990). Using the national networks: Bitnet and the Internet. Online 14, 24-29.

More specific than her article dealing with the information infrastructure (as per above), the author provides information about the major features available on both networks with basic and practical information about how and why one would use these two networks. This is useful information for the beginner because it explains the basic differences between Internet and Bitnet. Includes two sidebars on "Checking out the bulletin boards" and "Anonymous FTP: getting files on the Internet."

Communications, computers and networks: How to work and play and thrive in cyberspace. (1991). Scientific American [Special issue $] 265$.
This issue includes 11 articles, each written by an authority in the field, on how computer networking is changing business, education and even the way we work. Of particular interest is Michael Dertouzos' introductory article entitled "Communications, Computers and Networks" for its overview and discussion of the value of information and the essential components of an information infrastructure. Also notable is Lee Sproull and Sara Kiesler'sarticle "Computers, Networks and Work" which examines how the use of electronic mail changes patterns of communication within organizations.

Dern, Daniel.(1992). Applying the Internet:Corporate, research, educational, governmental, and other real-world uses. Byte. $17,111-115$.

Dern describes the Internet as "...evolving into the computer-based telephone/post office/CB radio/water cooler of tomorrow." He explores its many uses and provides an overview of the scope and range of applications on the Internet. Examples cited include scientific experiments facilitated by supercomputers on the Internet, locating files and software using Archie, searching OPACs, using the Internet as a teaching tool in the schools, and Internet uses in the business community.

Kalin, Sally and Tennant, Roy. (1991). Beyond OPACS... the wealth of information resources on the Internet. Database 14, 28-33.

The authors present a selection of information resources, such as network information center, formal and informal lists and resource guides, which help users navigate the Internet. This item is also interesting because of the authors' examination of issues such as evaluating the quality of a resource, access concerns, the need for ethical behavior and support and training issues. The article includes a sidebar on Specialized Resources which covers regional information, databases in the humanities, sciences and social sciences and software archives.

Notess, Greg R. (1992). Gaining access to the Internet.Online 16, 27-34.

Although originally limited to researchand academicspheres, access to the Internet is expanding into many different sectors. This article is of particular interest to those who currently do not have access to the Internet through their institution. It provides a table of commercial Internet providers current as of May 1992. This table includes the name, address, telephone and e-mail addresses of each provider. In addition information about the area served and prices charged by each provider is listed. The article discusses the different pricing schemes, the different levels of access to the Internet, and the different ways of obtaining access. Any potential restrictions are also covered. It also includes two sidebars entitled What to do once you're on the Internet and Freenets. 
Polly, Jean Armour. (1992). Surfing the Internet: An introduction. Wilson Library Bulletin 66, 38-42, 155.

Presented in an informative and readable manner, this article briefly describes the author's use of the Internet. It subsequently breaks down into a number of sections dealing with: electronic serials, remote login and access to resources, tools on Internet, information on how to connect, and further resources. Each section includes a brief explanation accompanied by a brief description of items of interest to the library community.

Quarterman, John S. (1990). The Matrix: Computer networks and conferencing systems worldwide. Bedford, MA: Digital.

This is one of the most frequently cited works in the literature on networks. It provides a broad overview of networks and conferencing systems, their use, their mechanics and their interconnection. The first half of the work is dedicated to introductory explanations of basic terminology and services, network mechanics and protocols and historical information. The second part describes specific networks and conferencing systems and is arranged geographically to facilitate consultation. Described by the author as a "random access book" it is useful as a reference guide and text for ongoing consultation. It includes extensive bibliographies after each chapter and a detailed index. The author is now the publisher of The Matrix News, a monthly newsletter which examines ongoing developments in the field.

\section{INTERNET APPLICATIONS}

\section{Electronic Mail}

Bailey, Jr., Charles W. (1991). Electronic (online) publishing in action ... The Public-Access Computer Systems Review and other electronic serials. Online 15, 28-35.

The author describes how the PACS-L (Public Access Computing listserv) computer conference expanded into an electronic newsletter, and an electronic serial entitled The Public-Access Computer Systems Review, one of the first electronic serials for the library community. The discussion ranges from technical aspects, such as how to format and distribute this kind of material to reflections on some of the key issues involved. This item has been included because PACS-L is one of the most popular electronic discussion groups for inf ormation professionals and its publications are equally of interest. A sample page from the Review is included. There are sidebars devoted to Conferences on Bitnet and Electronic Serials on Bitnet.

Bailey, Jr., Charles W. (1991). Library-oriented computer conferences and electronic serials on Bitnet and Internet. Database Searcher 7, 22-23.

The authorprovides basic information about what computer conferences are and their utility. This item is particularly useful because it contains a table of conferences of interest to librarians with their respective electronic mail addresses. It also includes simple specific instructions on how to subscribe. Includes short bibliography.

Bailey, Jr., Charles W. (1992). Network-based electronic serials. Information Technology and Libraries 11, 29-35.

This is a thoughtful discussion by a pioneer in the field about how electronic publishing will effect scholarly communication. The article examines the emergence of network-based electronic serials, provides brief descriptions and specific titles of electronic newsletters (citing 6 titles of interest to librarians) and electronic journals (citing 7 titles of more general interest) and concludes with a listing of 12 issues which remain to be resolved in this new area.

Delfino, Erik. (1990). E-Mail connections: It's still a jungle out there ...but it is getting better. Online 14, 31-35.

Although somewhat dated now, this article is interesting because it discusses a number of e-mail systems (BITNET, CompuServe, DIALMAIL, MCI Mail, Internet, and the now defunct ALANET) and explains each one's addressing system. The article briefly discusses the lack of a global directory and suggest that the "best tool for getting this information [another user's e-mail address] from your correspondents is still (gasp!) the good ol' telephone, or heaven forbid, paper mail." Includes sidebars on How to reach other e-mail systems and Electronic mail systme customer service departments.

Goode, Joanne and Johnson, Maggie. (1991). Putting out the flames: The etiquette and law of e-mail. Online 15, 61-65.

Because it lacks visual or aural clues, it is important to understand the dynamics of e-mail etiquette. In the first part of the article the authors present guidelines on style, mail management and general courtesy. Privacy is the focus of the second part of this item with a discussion of the need for privacy, legal issues and future developments in this area.

\section{Remote Login}

Farley, Laine, (Ed.). (1992). Library resources on the Internet: Strategies for selection and use. RASD Occasional Papers, Number 12. Chicago, IL: Reference and Adult Services Division, American Library Association.

This slim guide is specifically designed to facilitate consultation of OPACs. After a brief discussion of the benefits of using OPACs, the authors then turn to providing technical tips on how to use the various systems successfully. Guidelines for search strategies are included. In addition to a brief annotated bibliography, there are several appendices. One, in particular, includes sample menu screen and instructions by function for six major vendors as well as homegrown systems. 
Nickerson, Gord. (1991). Remote Log-in with Telnet. Computers in Libraries 11, 25-26.

This is a practical discussion of what the telnet application does and the potential terminal problems that a user might encounter. The author includes a sample session which is useful to first time users.

Notess, Greg R. (1993). The Internet meets online. Online 17, 8486.

This is the first article in a new column which will be appearing in Online and Database on the subject of the Internet. As such it includes a short review of articles already published in Online and Database, an update of more commercial Internet access providers not mentioned in the author's article "Gaining access to the Internet" published in Online in 1992. Six actual questions which can be answered using the resources on the Internet are presented. They illustrate the breadth of coverage available.

Tenopir, Carol. (1992). Online searching with Internet. Library Journal XX, 102-104.

This item is useful to those whowould like to use Internet to search commercial online systems. Following a description of the main Internet applications, the author presents a table of Internet addresses for vendors with their associated costs per hour.

\section{File Transfer}

Nickerson, Gord. (1991). File transfer protocol. Computers in Libraries 11, 51-53.

Recommended for basic advice and simple explanations, this brief article provides a short table of the major commands to use and their utility. It includes a sample of an anonymous FTP session and a list of FTP sites. The author discusses the archive sites where much of ftp material can be found and the problems you may encounter.

Tomer, Christinger. (1992). Anonymous FTP resources. Academic and Library Computing 9,8-12.

After a brief description of FTP, the author provides a number of tables depicting the most popular archive sites for obtaining files and sof tware. This article alerts readers to particular sites and briefly profiles some of the more popular sites.

\section{TOOLS ON THE INTERNET}

\section{Archie}

Deutsch, Peter. (1992). Resource discovery in an Internet environment-the Archie approach. Electronic Networking 2, 4551.

Written by one of the architects of Archie, this article begins with a discussion of the "so-called Resource Discovery Problem". It explains how Archie was created in response to the "problem of indexing anonymous FTP archives". Coverage includes how to access and use the Archie service. This item also describes future developments are planned for Archie.

Simmonds, Curtis. (1993). Searching Internet archive sites with Archie: Why, what, where and how. Online 17, 50-55.

In addition to basic information on the functions Archie performs, its advantages and a list of Internet Archie server addresses, the author presents detailed information on three different methods that can be used to access it: via telnet, using local client sof tware and via e-mail. The article also includes a discussion of Gopher as it relates to Archie and a description of the Whatis database used in conjunction with Archie to locate files through subject keywords.

Gopher

The Internet Gopher: An information sheet. (1992). Electronic Networking, 2, 69-71.

This concise fact sheet provided by the University of Minnesota Gopher Development Team describes Gopher as "an information distribution system" which allows users to browse, search and store information from a wide variety of sources. It includes basic information on what's available, how Gopher works, and how it is accessed. For those interested, there is additional information on who to contact, electronic discussion group addresses, etc.

Notess, Greg R. (1993). Using Gophers to burrow through the Internet. Online, 17, 100-102.

The author suggests that even if you use the Internet infrequently, Gopher is one of the tools to use to navigate it effectively. The author provides a basic explanation of what it is, how it works and a list of suggested locations. Shortcomings are also discussed as well as one of the latest tools on the network, Veronica. 
Kahle, Brewster, and Medlar, Art. (1991). An information system for corporate users: Wide area information servers. Online 15, 56-60.

Co-authored by Brewster Kahle, a pioneer in WAIS development, this item is interesting for its explanation of how Wais was first developed out of a project involving Dow Jones, Thinking Machines Corp., Apple Computer and KPMG Peat Marwick. WAIS eliminates the need for the user to be familiar with a number of different systems because it has the capacity to search a variety of information resources and simply present the results to the user. This article discusses the rationale for developing this server. There are a number of illustrations which demonstrate how a user can pose a question and what kind of results are obtained.

Lukanuski, Mary. (1992). Help is on the WAIS. American Libraries ??, 742-744.

After a brief description on how WAIS was developed, the author provides a clear explanation of how it works which covers the question box, the source box and the answer box. Analysis of the advantages and disadvantages of Wais is also included.

Nickerson, Gord. (1992). Getting to know wide area information servers. Computers in Libraries 12, 53-55.

This brief article succinctly explains how WAIS was developed, how it works, its advantages and its utility as well as furnishing suggestions on how to improve it. The author also provides a specific address with instructions for users to experiment with WAIS.
The amount of information available in print about Internet is increasing. Several years ago most of the guides to the Internet were available as electronic documents which were obtained using FTP. The increase in guides alone, published in the last year or two, is testimony to the increasing popularity of the Internet and the need for accessible documentation. As the number of applications on the Internet multiplies and its use in many sectors of society increases, one can safely predict that the volume of information will increase accordingly in a variety of formats. This increase in print is advantageous because it expands awareness about the Internet beyond those already using it. However, the major disadvantage of print is generally its lack of currency. This is a major handicap when dealing with a topic as dynamic as the Internet. Many of the items cited in this bibliography, in particular the monographs, may be in the process of revision and more recent editions may now be available.

For those who would like to follow further print sources, several journals offer regular columns. For example, Computers in Libraries has regular sections entitled Networked Resources and Electronic Publishing which frequently deal with the Internet. The sister publications, Online and Database launched a new column in March 1993 entitled "On the nets" which will deal with Internet. Finally, two new journals are suggested. Electronic Networking: Research, Applications and Policy, begun in 1991 , is devoted to the discussion of issues dealing with electronic networking and computer networks. Internet World, formerly entitled Research \& Education Networking, is a new monthly publication which will provide news, interviews, and analyses of Internet developments.

\section{ERRATA}

Additional information: v.16, no. 3

Bibliography attached to the article by Dr. John U. Ogbu (pp.12-13) and Bibliography to the article by Dr. Bernard R. Gifford

Both these bibliographies were compiled by:

Maxine Goldsmith

New Jersey Dept. of Higher Education

Chair, Education Division 1992/93 